\title{
The effect of bundling medication-assisted treatment for opioid addiction with mHealth: study protocol for a randomized clinical trial
}

David H. Gustafson Sr${ }^{1}$, Gina Landucci ${ }^{1}$, Fiona McTavish ${ }^{1}$, Rachel Kornfield ${ }^{1,2}$, Roberta A. Johnson ${ }^{1 *}$, Marie-Louise Mares ${ }^{3}$, Ryan P. Westergaard ${ }^{4}$, Andrew Quanbeck', Esra Alagoz', Klaren Pe-Romashko', Chantelle Thomas ${ }^{5}$ and Dhavan Shah ${ }^{6}$

\begin{abstract}
Background: Opioid dependence has devastating and increasingly widespread consequences and costs, and the most common outcome of treatment is early relapse. People who inject opioids are also at disproportionate risk for contracting the human immunodeficiency virus (HIV) and hepatitis $\mathrm{C}$ virus (HCV). This study tests an approach that has been shown to improve recovery rates: medication along with other supportive services (medication-assisted treatment, or MAT) against MAT combined with a smartphone innovation called A-CHESS (MAT + A-CHESS).

Methods/design: This unblinded study will randomly assign 440 patients to receive MAT + A-CHESS or MAT alone. Eligible patients will meet criteria for having an opioid use disorder of at least moderate severity and will be taking methadone, injectable naltrexone, or buprenorphine. Patients with A-CHESS will have smartphones for 16 months; all patients will be followed for 24 months. The primary outcome is the difference between patients in the two arms in percentage of days using illicit opioids during the 24-month intervention. Secondary outcomes are differences between patients receiving MAT + A-CHESS versus MAT in other substance use, quality of life, retention in treatment, health service use, and, related to HIV and HCV, screening and testing rates, medication adherence, risk behaviors, and links to care. We will also examine mediators and moderators of the effects of MAT + A-CHESS. We will measure variables at baseline and months 4, 8, 12, 16, 20, and 24. At each point, patients will respond to a 20- to 30-min phone survey; urine screens will be collected at baseline and up to twice a month thereafter. We will use mixed-effects to evaluate the primary and secondary outcomes, with baseline scores functioning as covariates, treatment condition as a between-subject factor, and the outcomes reflecting scores for a given assessment at the six time points. Separate analyses will be conducted for each outcome.
\end{abstract}

Discussion: A-CHESS has been shown to improve recovery for people with alcohol dependence. It offers an adaptive and extensive menu of services and can attend to patients nearly as constantly as addiction does. This suggests the possibility of increasing both the effectiveness of, and access to, treatment for opioid dependence.

Trial registration: ClinicalTrials.gov, NCT02712034. Registered on 14 March 2016.

Keywords: Technology, mHealth, Addiction, Medication-assisted treatment, Opioids, Smartphone, HIV, HCV

\footnotetext{
* Correspondence: bobbie.johnson@wisc.edu

${ }^{1}$ Center for Health Enhancement Systems Studies, University of

Wisconsin-Madison, Madison, WI 53706, USA

Full list of author information is available at the end of the article
}

(c) The Author(s). 2016 Open Access This article is distributed under the terms of the Creative Commons Attribution 4.0 International License (http://creativecommons.org/licenses/by/4.0/, which permits unrestricted use, distribution, and reproduction in any medium, provided you give appropriate credit to the original author(s) and the source, provide a link to the Creative Commons license, and indicate if changes were made. The Creative Commons Public Domain Dedication waiver (http://creativecommons.org/publicdomain/zero/1.0/) applies to the data made available in this article, unless otherwise stated. 


\section{Background}

Opioid dependence has devastating consequences for patients, family members, and communities. In 2012, an estimated 2.1 million Americans had opioid use disorders (OUDs) related to prescription opioids, and 467,000 had OUDs related to heroin [1]. The total volume of opioids prescribed in the health care system has risen steeply in recent years. In 1991, about 76 million prescriptions were written for opioids; in 2013, about 207 million prescriptions were written [2]. A growing proportion of people with OUDs started their use of opioids by taking prescription opioids. Emergency department visits related to the nonmedical use of opioids rose from 144,600 in 2004 to 305,900 in 2007 [3] and unintentional overdose deaths from opioids have more than quadrupled since 1999, reaching their highest level ever in 2014 $[2,4]$. OUDs also have been a primary driver of the increased spread of human immunodeficiency virus (HIV) and hepatitis $\mathrm{C}$ virus (HCV) in many rural and suburban communities in the US $[5,6]$.

Existing treatments for OUDs often fail. Following detoxification from opioid dependence, early relapse is the most common outcome [7]. After inpatient treatment, the vast majority of patients relapse within a year, often within the first few months [8]. Medication-assisted treatment (MAT) arose when methadone became available in the 1960s. Along with other supportive services, such as peer support, MAT has been shown to increase rates of recovery from OUD [9]. Yet those who receive MAT still do not generally maintain long-term abstinence $[8,10]$.

Access to treatment is an enormous challenge, with only $10.7 \%$ of OUD patients who needed treatment in 2012 receiving it [11]. Effective treatment is complex and demanding because OUDs are chronic diseases that require ongoing medication, behavioral counseling, and overdose protection, as well as screening and treatment for infectious disease and comorbid psychiatric disease [2]. Effective treatment is also complex because affected populations differ in the etiology and course of their addiction, motivation for treatment, and reasons for relapse, including notable differences between men and women [12]. For example, women tend to progress more quickly from the start of substance use to the start of dependence, have a higher rate of cooccurring mood and anxiety disorders, and have better outcomes on buprenorphine than on methadone [12]. Finally, retention in treatment, which is known to reduce drug use [13], remains a challenge in treating OUDs [14-19]-so much so that treatment retention is often itself regarded as a desired outcome [20].

Testing and links to care for HIV and HCV are essential for people who inject opioids. Those who inject drugs are at greater risk of contracting HIV and are less successfully linked to [21-23] and retained in [24-26] clinical care.
Antiretroviral therapy is recommended for all patients living with HIV, but treatment is under-used [27] and often suboptimally effective $[23,28]$ among people who inject drugs. HCV occurs primarily in people who inject drugs, with $90 \%$ of older injection-drug users infected [29-31]. $\mathrm{HCV}$ is the most common blood-borne infection in the US [32] and the most common cause of end-stage liver disease and the need for liver transplants.

The randomized clinical trial described here assesses the extent to which the considerable challenges of effectively treating OUDs can be addressed by an mHealth intervention. Specifically, we pair MAT with a smartphone-based innovation called Addiction CHESS (A-CHESS). A large $(n=349)$ randomized controlled trial (RCT) previously found that A-CHESS decreased risky drinking days and enhanced long-term abstinence among alcohol-dependent people leaving residential treatment, one third of whom reported illicit opioid use [33]. Related field tests in the Veterans Administration and drug courts and among pregnant women in Appalachia [34] also found a positive impact on alcohol and opioid abuse of providing smartphones with A-CHESS. In this trial, we assess the potential of A-CHESS to improve long-term outcomes of MAT among OUD patients. Furthermore, the study seeks to understand-through analyses of mediators and moderators and exploratory analyses-the ways in which A-CHESS works and does not work, for whom, and under what circumstances. Our research team has also developed and pilot-tested systems for improving engagement in care for patients with HIV and improving testing and links to care for patients with HCV. For the present study, these innovations related to HIV and HCV have been incorporated into A-CHESS, allowing us to evaluate whether A-CHESS can also improve screening and treatment outcomes for these conditions.

\section{Methods/design \\ Study design, hypotheses, and outcomes}

The study, a RCT, will assign 440 opioid users from three addiction treatment centers to receive either MAT + A-CHESS or MAT alone. Patients will be followed for 24 months. The primary hypothesis is that participants assigned to MAT + A-CHESS will have, compared with a control group, a lower percentage of days using illicit opioids. Secondary hypotheses are that those assigned to MAT + A-CHESS will have, compared to the control group, less use of other nonprescribed substances, higher quality of life, greater retention in treatment, and lower health service use. Secondary hypotheses related to $\mathrm{HIV} / \mathrm{HCV}$ are that those assigned to $\mathrm{MAT}+\mathrm{A}-$ CHESS will have higher screening and testing rates, greater medication adherence, fewer risk behaviors, and better linkage to care (i.e., referrals that result in in- 
person visits with providers). We also hypothesize that autonomy (or intrinsic motivation), competence, and relatedness [35] will mediate the effect of MAT + A-CHESS, along with negative affect and self-stigma. We will also determine the person-level factors that moderate the impact of MAT + A-CHESS versus MAT alone (e.g., gender, SUD severity, pain severity, severity of withdrawal symptoms, and loneliness). For patients receiving MAT + A-CHESS, we will examine whether patterns of using A-CHESS and communication style within peer discussion forums are predictors of study outcomes [36]. Figure 1 shows the logic and outcomes for the project. We will use quantitative and qualitative analyses to examine long-term impact, with survey data collected every 4 months during the 24-month period.

\section{Interventions}

\section{Control condition: MAT}

Patients in the control condition will receive treatment as usual including MAT. Treatment could consist of a recovery plan, medication, and regularly scheduled behavioral interventions such as monthly group counseling sessions, sessions with a substance abuse counselor, and Narcotics Anonymous/Alcoholics Anonymous (NA/AA) meetings. Medication may include methadone, injectable naltrexone, or buprenorphine. The sequence and duration of medication and behavioral interventions will vary by patient and site. Discussions with sites revealed that we should not try to control variations because they are tailored to individual patients. We will document which medications are used and will include as covariates when and how medications change between the 4-month surveys.

\section{Experimental condition: MAT + A-CHESS}

Patients in the experimental condition will receive a smartphone with A-CHESS for 16 months along with MAT as described above. A-CHESS is designed to improve recovery from addiction. A-CHESS is based on self- determination theory (SDT), which holds that meeting three needs-for autonomy, competence, and relatedness-improves a person's adaptive functioning [35, 37]. Figure 2 shows how A-CHESS services relate to the constructs of SDT and to the determinants and antecedents of relapse identified by Marlatt [16, 38, 39]. A-CHESS services provide antecedent-appropriate intervention(s) that boost autonomy (intrinsic motivation) by selecting from multiple services those most likely to be most personally meaningful to the patient; offer information, monitoring, and tools to increase competence; and/or increase relatedness. For example, the lower part of Fig. 2 shows antecedents of relapse, one of which is lifestyle imbalance (lower left of figure) and Marlatt's suggestion that developing substitute indulgences helps (second level-left). The left upper section shows how A-CHESS helps. Another example: A-CHESS monitoring tools include a weekly check-in and GPS-based tracking to identify when lifestyle imbalance may place a patient at risk of using drugs or engaging in unsafe sex. As one healthy alternative, the A-CHESS healthy events calendar may suggest one of the patient's healthy pleasures, such as going for a walk, and offer a map. We anticipate that this just-in-time approach may be important to help maintain abstinence. Figure 3 shows the A-CHESS user interface. Key A-CHESS services are described below.

Help. When a patient presses Help, the system shows a list of the patient's preapproved supporters and their phone numbers so the patient can easily call for help. The patient can also be linked to positive and potentially distracting activities such as selected games [40] and audio/video-based relaxation recordings [41].

Cognitive behavioral therapy (CBT) boosters offer brief, easy-to-remember reviews of CBT skills that patients learned during treatment to prepare them for future challenges-e.g., how to handle urges and anticipating, avoiding, and mitigating the effects of high-risk people, places, and things related to past drug use.

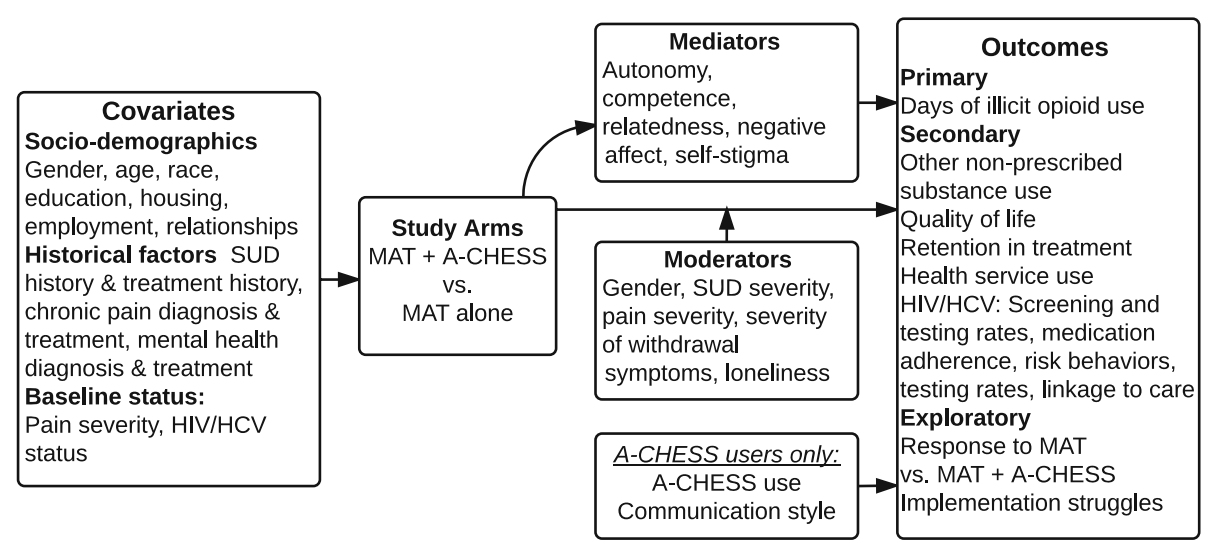

Fig. 1 Logic and outcomes of the study 


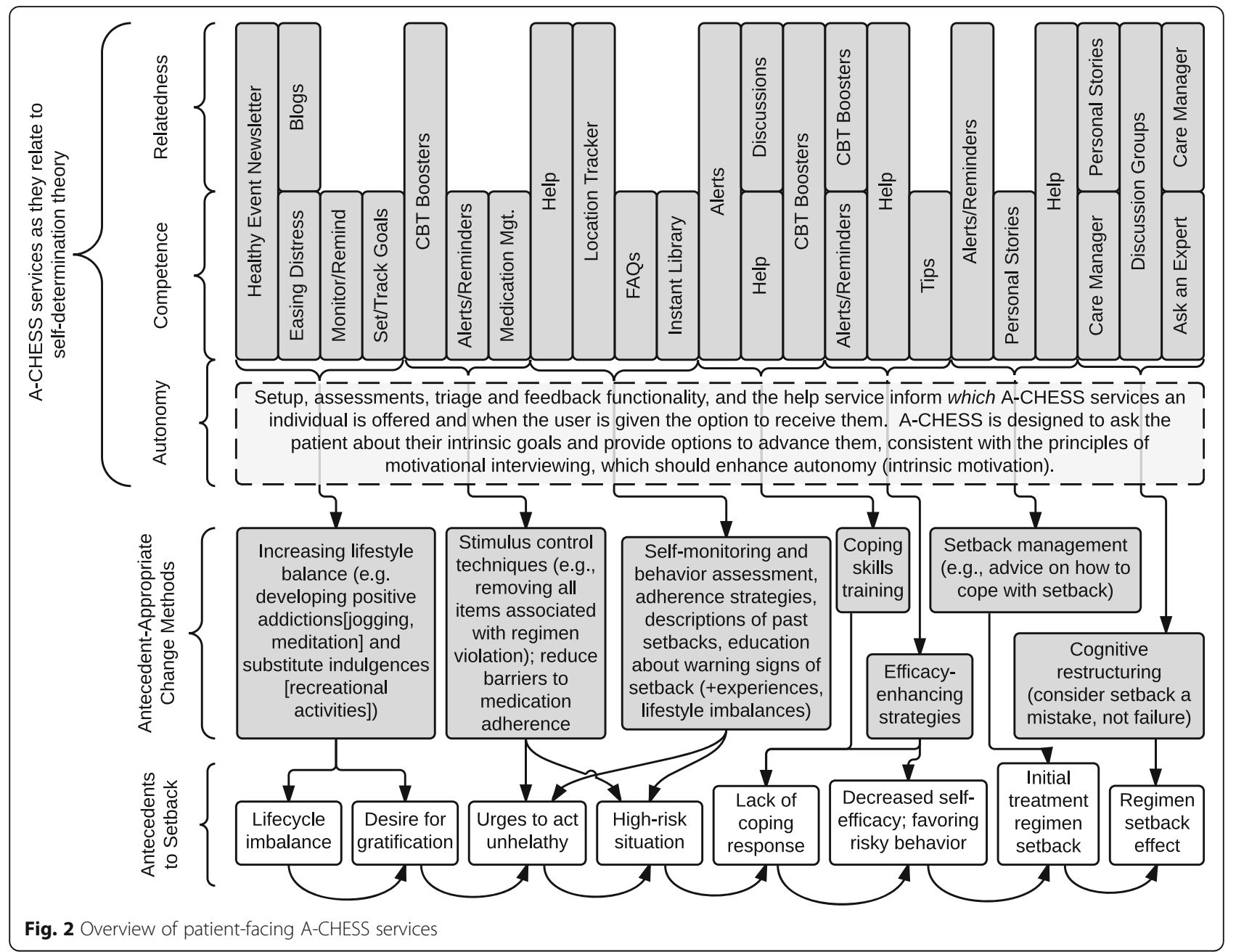

Monitoring functionality includes the location tracker (described below), self-assessment tools, and a record of A-CHESS use. One self-assessment tool is the Brief Addiction Monitor (BAM) [42], which we implement as a weekly survey. After completing the BAM, participants receive tailored feedback that acknowledges their use of protective behaviors over the past week and provides recommendations for addressing risky behaviors, including links to appropriate A-CHESS content. Participants reporting opioid or other drug use will be encouraged to seek appropriate help.

The location tracker uses GPS to monitor patient movement. If a patient approaches a location that they previously defined as high risk, A-CHESS will initiate a patient-defined recovery process. This might start with a beep, then a vibration, and then a list of preapproved contacts and options for distraction or mindfulness. The GPS service is also often used to find a 12-step meeting. Patients may turn off the location tracker if they perceive certain services to be too burdensome or invasive.
Triage and feedback functionality is designed to derail the relapse process, giving the patient just-in-time, tailored coping support. A-CHESS will be customized at the start by each patient to set options that will be triggered in a moment of need. For example, if a patient reports a craving triggered by environmental cues, such as seeing someone else use, A-CHESS might remind the patient of relaxation exercises, connect them to online peer support and the healthy events calendar, and/or notify a counselor, who may initiate contact via text or private message. Participants whose patterns of using A-CHESS demonstrate they are likely to stop using the system will receive automated messages and tailored messages from coaches to encourage them to reengage.

The counselor dashboard [43], developed by addiction physicians and psychologists, harvests clinically relevant data from A-CHESS and presents it to counselors to help them quickly: (1) identify patients who may be at high risk for relapse and/or benefit from clinical intervention, (2) see a detailed analysis of a patient's recent 


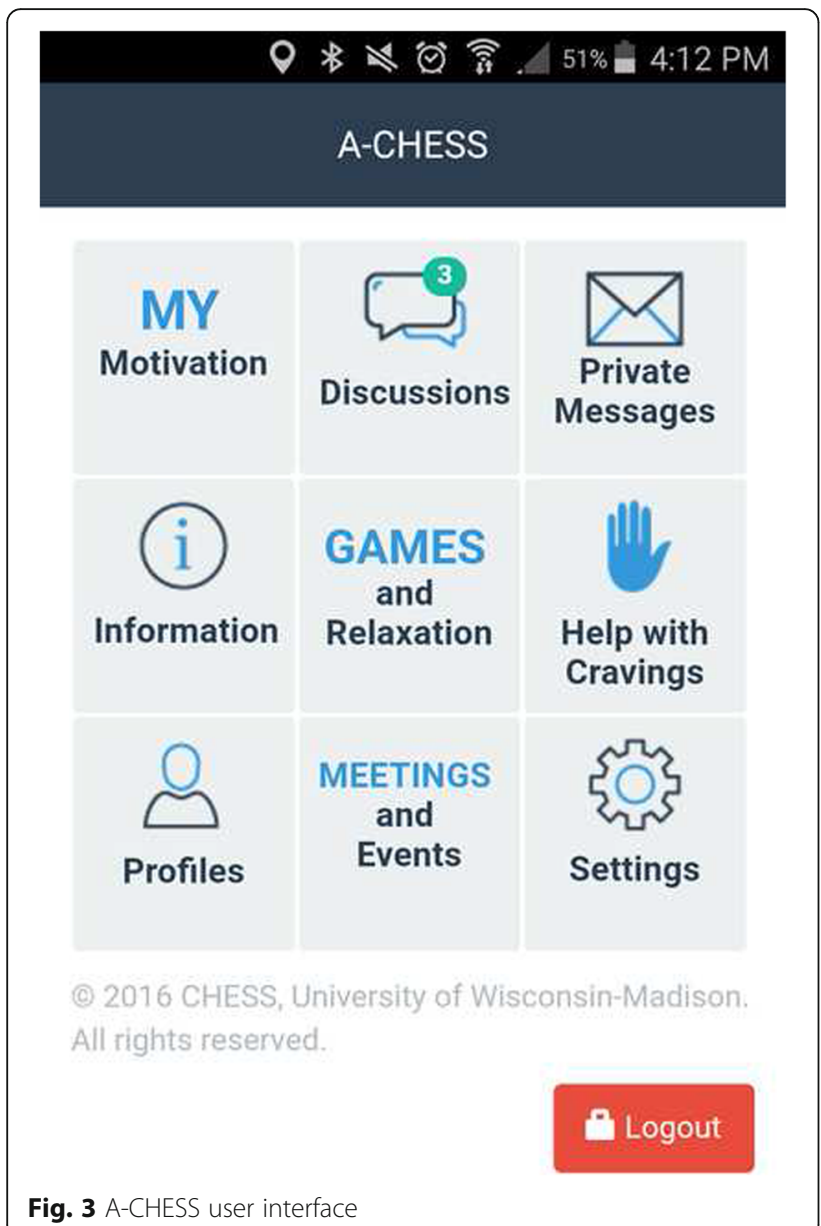

history, e.g., trends in individual BAM items, A-CHESS use, and relapse data, and (3) intervene with patients (e.g., through texting in A-CHESS). When a counselor logs into the counselor dashboard, he sees 'red pins' generated when A-CHESS (using counselor-determined priorities) detects that a patient may be at high risk. The counselor can adjust the cutoffs for red pins so the ones he sees are most useful.

HIV/HCV services. A-CHESS will integrate components of our team's existing computerized risk reduction systems that collect data on patients' $\mathrm{HIV} / \mathrm{HCV}$ risk behaviors and deliver behavior change interventions tailored to the patient's self-reported readiness for change. At enrollment, participants in both study conditions will be asked if they have been screened for HIV and HCV. Patients who test negative or decline testing at baseline will be sent reminders from A-CHESS about future testing at a frequency based on reported risk behaviors. Patients found to be HIV or HCV-positive will be provided with targeted multimedia health education content, access to online resources, and location-specific links to clinical care and case management.
Coach-monitored discussion groups [44] foster the exchange of emotional, informational, and instrumental support among patients. Discussions are monitored daily by an A-CHESS coach to encourage appropriate use. Coaches are not counselors, but are members of the research team trained on A-CHESS, risk identification, referral, and technology-based patient engagement. They are skilled in constructive interaction and persistence and are willing to work unusual hours. The coaches encourage individuals to follow up with their health care providers/prescribers regarding medication-assisted treatment questions. We found that a coach increases and sustains use of A-CHESS [45]. Every week, a coach reviews use data. Based on what the coach sees, they write messages to the participants. For example, (1) to a patient active on A-CHESS, "Hi kfields05, Just wanted to say hello and see how things are going. Looks like you are doing a great job of recovery and tracking, which is wonderful. Let me know if there is anything I can help with. Take care and keep it up! Coach Lola." and (2) to a patient who is not logging in: "Hi Teresa $\mathrm{H}$, Just checking in to see how you are coming with your recovery goals. You have not logged in for a while so I figured I would say hi. Take care and let me know what I can do to help! Coach Lola."

Possible counselor alerts. A-CHESS sends an email notification to an A-CHESS coach if a patient reports substance use or is over a preset risk threshold on selfmonitoring items. The coach may alert a counselor or encourage the patient to seek further support within A-CHESS (e.g., by using discussion groups, games, and relaxation exercises; revisiting their personal recovery motivation; or listening to personal stories from others in recovery) or recommend that the patient seek other professional help.

\section{Ethics}

The study received approval from the Health Sciences Institutional Review Board at the University of Wisconsin-Madison (\#2015-1418) and the Western Institutional Review Board (\#1163410) in Puyallup, Washington and is registered at ClinicalTrials.gov (NCT02712034). The study complies with the relevant Standard Protocol Items: Recommendations for Interventional Trials (SPIRIT) Statement and World Health Organization Checklist (see the SPIRIT Checklist and figures in Additional files 1 and 2). The study is funded by the United States Department of Health and Human Services National Institute on Drug Abuse.

\section{Patient eligibility}

Patients will be recruited from outpatient detoxification and treatment programs at three sites, two in Massachusetts and one in Wisconsin. Patients are eligible for 
the study if they (1) are currently on MAT (methadone, injectable naltrexone, or buprenorphine) for their substance use disorder (SUD), (2) are aged 18 years or older, (3) meet criteria for having an OUD of at least moderate severity (4 or 5 Diagnostic and Statistical Manual of Mental Disorders, fifth edition (DSM-V) criteria), (4) have no acute medical problem requiring immediate inpatient treatment, (5) have no history of psychotic disorders, though patients with other comorbid psychopathology (mood disorders, anxiety, other substance use disorders) will be eligible, (6) are willing to participate in a randomized clinical trial, (7) provide the name, verified phone number, and address of at least two contacts willing to help locate the patient, if necessary, during follow-up, (8) are able to read and write in English, (9) are not pregnant, (10) are willing to share health-related data with primary care clinicians, and (11) are, at study intake, abstinent from opioids for at least 1 week and no longer than 2 months, except for medications used to treat the disorder.

\section{Recruitment}

Potential subjects will be identified by a staff person at each of the three sites and asked if they are interested in learning about a study for which they may be eligible. If they answer yes, the University of Wisconsin (UW) or site coordinator will provide a detailed overview of the study, including patient responsibilities and how patient confidentiality will be protected. Interested patients will then provide informed consent, complete a baseline survey, be randomized to receive MAT + A-CHESS or MAT, and, if applicable, be trained on A-CHESS. Figure 4 shows the flow of participants through the trial.

\section{Randomization}

The project director will use a computer-generated allocation sequence to randomize participants in a 1:1 ratio to MAT + A-CHESS or MAT alone, stratifying on gender and site and balancing on age, level of care (intensive outpatient treatment, day treatment, or weekly or monthly counseling), and whether patients had prior SUD treatment. The project director will inform the site coordinator of the group assignment by email; in the email, the participant will be identified only by study ID (the code used to make the identity of participants unknown). The site coordinator will initiate patients into the study condition and, if the patient is assigned to MAT + A-CHESS, provide training.

\section{Smartphone distribution}

Patients randomized to MAT + A-CHESS who do not already have an Android smartphone will be given one

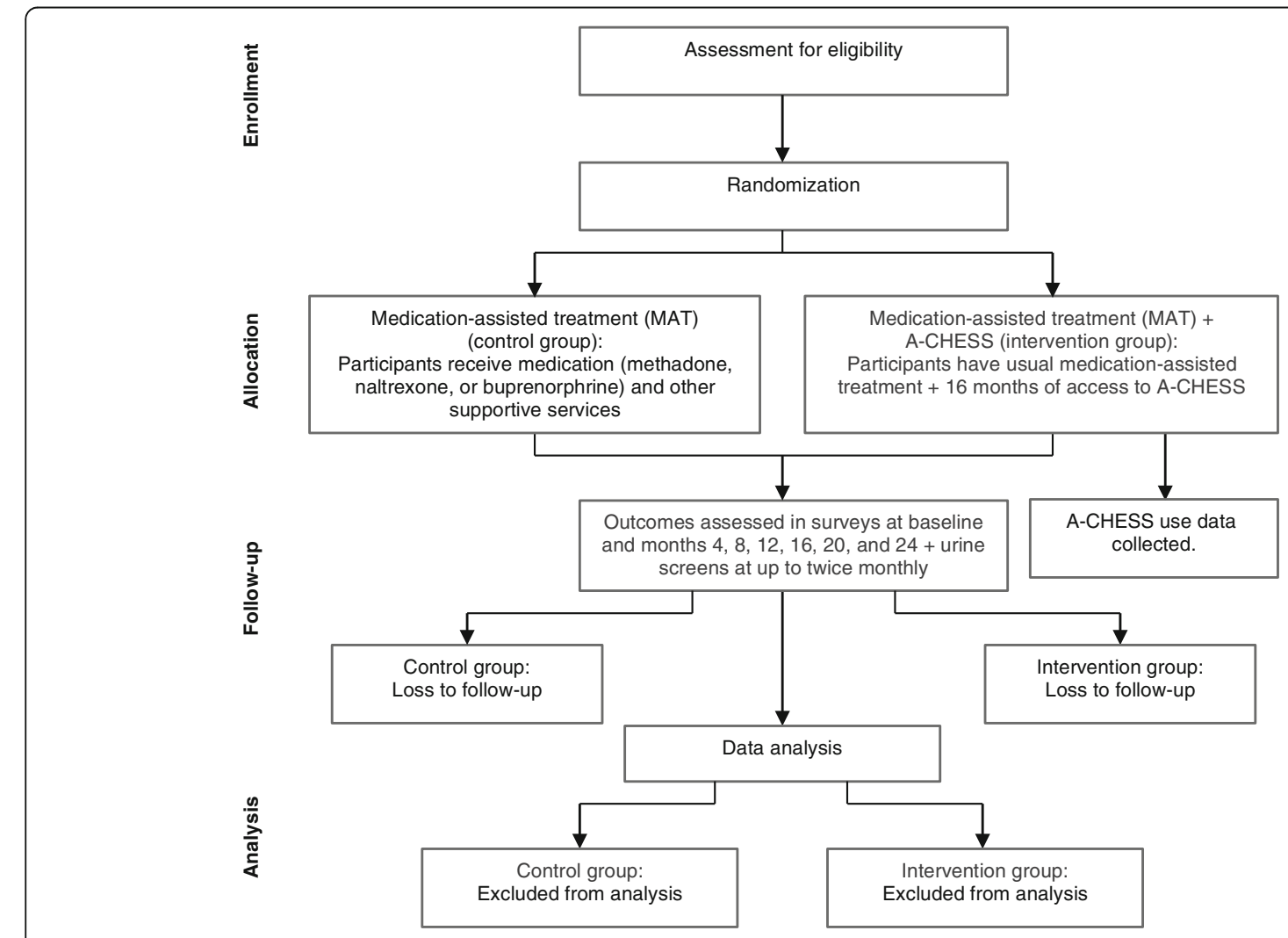

Fig. 4 Participant flow 
loaded with A-CHESS, along with a data plan that includes unlimited data, text, and voice for 16 months. Patients who already have an Android smartphone will have A-CHESS installed on their phone. We will provide up to one replacement phone to patients who report their phone lost, stolen, or broken. If patients lose the second phone, we will offer to load A-CHESS onto an appropriate replacement smartphone (e.g., Samsung S5) that they obtain. We have included in the budget a $20 \%$ allowance for replacement phones, which has proven sufficient in prior CHESS research, including trials with populations of addicted patients [33, 46].

\section{Training to use A-CHESS}

The UW or site coordinator will train patients to use the A-CHESS app and customize it - e.g., by sources of support (such as family), contacts who detract from recovery (such as friends who use illegal drugs) and support recovery, and so on. A-CHESS will be updated monthly with activities for the healthy event calendar; changes, if any, to therapeutic goals and the recovery plan (e.g., self-help groups, medication) and in home, work, or educational responsibilities; and high-risk locations to avoid. Patients must demonstrate that they can use A-CHESS (e.g., make one post to the discussion group) before they leave the training session with the phone.

\section{Quantitative data collection}

All subjects will complete follow-up surveys over the phone with the UW study coordinator at months $4,8,12$, 16,20 , and 24. Data collected will relate to the variables shown in Fig. 1. Each phone survey is expected to take 20 to $30 \mathrm{~min}$. Surveys will be identified by study ID, not participant name. The form linking study IDs and names will be kept in REDCap [47]. In addition, urine screens, which are done routinely as part of MAT, will be conducted at each study site and recorded for all subjects at baseline and up to twice a month thereafter. Results will be used to validate self-reported information. Inconsistent results between urine drug tests and self-report results will not affect patients' ability to continue in the study.

\section{Qualitative data collection}

We will administer in-depth interviews with patients to shed light on their perspectives as well as on what promotes and hinders implementing and sustaining MAT + A-CHESS. We will explore patient perceptions of the effects of integrating MAT + A-CHESS, the most and least useful services in A-CHESS, gender-specific effects, and how patients feel about various A-CHESS services over time. A second set of interviews will examine provider perceptions of benefits of and barriers to integrating and sustaining MAT + A-CHESS over time.
A third set of interviews will examine fine-tuning MAT + A-CHESS; communication between the research team, patients, and providers; and concerns from providers and patients. These data will help to refine methods for developing mHealth systems generally.

UW research staff will also conduct a longitudinal case study of five female and five male patients to explicate MAT + A-CHESS effects, considering patients' medical and addiction treatment history, family history, personal and gender-specific preferences, and environmental factors. Case studies, though underused in health care [48], are a good way to understand how innovations work in real life [49]. They provide insight into patterns that might be overlooked in RCTs because they reveal the complexities of systems in which innovations are introduced. By following these ten individuals over time, we will explore how women and men integrate new technologies into their lives, circumstances that favor or complicate the process, and barriers to sustainability. Data collection from interviews and focus groups will ensure the comprehensiveness of findings and strengthen validity [50].

\section{Measures}

All scales have good psychometric properties with similar populations. Listed below are the factors to be measured and instruments to be used, along with references to validation studies for the instruments.

Intake and baseline. Treatment center staff will document patient eligibility. Then patients will complete the baseline survey. They will report 12 demographic items (gender, age, race, education, etc.) and five items related to their opiate use history (age of first use, age of regular use, whether opioid use began with a physicianprescribed opioid, number of past quit attempts, and date of last use). Patients will also respond to items related to chronic pain diagnosis and treatment, current and past comorbid psychiatric diagnoses and treatment, pain severity (using the Numeric Rating Scale (NRS-11)) [51], and HIV/HCV status.

Dependent variable. Self-reported illicit opioid use days will be analyzed in 30-day periods. At baseline, a Timeline Followback (TLFB) [52] for the 30 days before admission will be obtained (with opioid use separated from other drug use) and a urine drug screen collected (CTN-approved drug use outcome measures). For follow-up assessments, the TLFB for the previous 120 days will be obtained. The TLFB has been successfully used to obtain drug use data for extended periods of time and with polydrug-using patients [53].

At months 4, 8, 12, 16, 20, and 24. During phone surveys after baseline data collection, patients will complete a 120-day TLFB [54] to document their illicit opioid and other nonprescribed drug and alcohol use as well as health service use during the past 4 months. Health 
service use will be collected for the categories listed under "Health service use and cost" below. Patients will also complete measures of: relapse risk (Brief Addiction Monitor [55], nine items); pain severity (NRS scale [56], one item); HIV/HCV screening and link-to-care status (testing status and if tested, result, and if positive, whether the patient saw a medical provider); risk behaviors (HRBS [57], five items); status of current housing (two items) and employment (three items on type of employment, hours worked); quality of life (The Satisfaction with Life Scale [58], eight items); rating scale for withdrawal (three items); self-reported medication adherence (Morisky Medication Adherence Scale: MMAS-4 [59], four items); and loneliness (three items). We will collect number of phones lost, stolen, or broken (research records). For retention in treatment, we will take the proportion of appointments attended from clinic records. We will also determine if participants are engaging in other forms of treatment outside of the treatment facility, such as seeing a therapist, working with a sponsor, or attending NA/AA meetings.

Mediators. Self-determination theory (SDT) constructs will be assessed as follows: autonomy, Treatment Self-Regulation Questionnaire [60] (six items); competence, revised Drug-Taking Confidence Questionnaire [61] (eight items); and relatedness, our own bonding scale (five items). Negative affect will be assessed by Positive and Negative Affect: PANAS [62, 63] (20 items), and self-stigma by the self-devaluation subscale of the selfstigma in substance abuse scale [64] (seven items).

Moderators: we will focus our evaluation on gender but also collect data on other potential moderators (SUD severity as determined by the treatment site at intake using DSM-V criteria, pain as determined by the NRS-11, withdrawal, and loneliness).

A-CHESS use. A-CHESS use will be collected in timestamped log files and includes when a patient accessed A-CHESS, service(s) selected, duration of service use, pages viewed, messages posted versus received, and communication style and content of messages. Content will be subject to computer-automated content analysis to identify communication styles that may predict study outcomes. Cumulative use (number of pages viewed and days used) significantly predicted risky drinking days in the randomized trial of A-CHESS with alcohol- dependent patients [33]. We will also collect data on sources of other SUD-related information and support.

Health service use and cost. Our cost analysis is motivated by the potential that A-CHESS has shown to reduce the use of costly health services associated with relapse. (In a field test with US military veterans, A-CHESS users had substantially decreased rehospitalizations related to relapse.) Our approach to measuring and analyzing health use data is adapted from McCollister and French's 2003 analysis of the economic benefit of addiction interventions [65]. We will use the following categories of health service use: emergency room visits, hospital detox (day), and short-term residential treatment (day). We will also track costs for all other hospital visits and stays (in addition to emergency room visits and hospital detox); urgent care visits for any reason (to which we will apply cost estimates derived from a national survey of urgent care clinics [66]; individual psychotherapy or psychiatric care; and self-reported outpatient addiction treatment services after relapse (using the categories of outpatient addiction care outlined by McCollister and French [64], to which we will apply cost estimates provided in the 2008 national survey of 110 substance abuse treatment programs by French et al., adjusted for inflation [67]). We will also collect selfreported use of other health services (e.g., dental care, primary care, chiropractor), following the approach by Bell et al., to assess the cost-effectiveness of supervised versus unsupervised buprenorphine-naloxone administration [68]. We will derive cost estimates for various types of health care use (e.g., emergency room visits, hospitalizations, primary care visits, etc.) using data from the American Hospital Association and the American Medical Association.

\section{Timeline}

Recruitment began in April 2016 and will end in February 2018. The intervention period will end in February 2020. Figure 5 shows the study timeline.

\section{Data analysis \\ Power and sample size justification}

Primary analysis. Our study will be powered to detect a difference between MAT + A-CHESS and MAT in

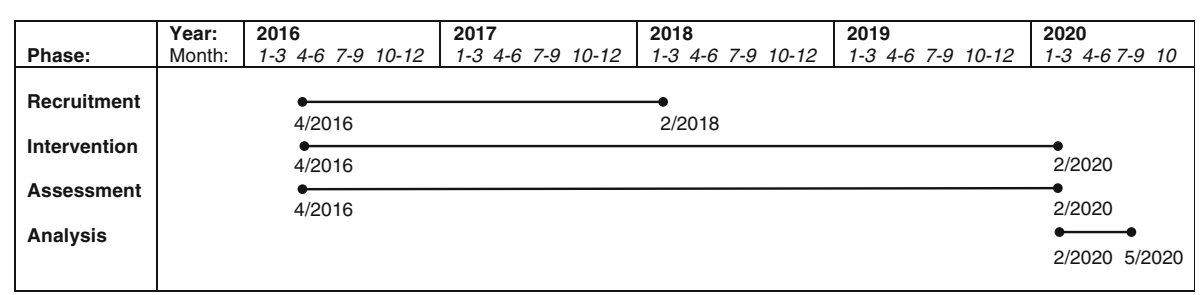

Fig. 5 Study timeline 
percentage of days using illicit opioids across the 24month intervention period. Based on data from the two sites from which most study participants will be recruited, we assume $35 \%$ attrition over the course of the study, providing an $N$ of 286 . Using the power program by Hedeker et al. [69] and assuming up to cubic trends in the data, given expected attrition, recruitment of 440 patients will provide $80 \%$ power to uncover a standardized difference of .35 across the 24 months. From prior data [70,71], this would be a difference of approximately three opioid use days/month depending on the observed standard deviation.

Secondary analyses. Power for examining intervention effects for the secondary outcomes would be similar as for the percentage of opioid use days, though the difference implied by the standardized difference of .35 will depend on the actual standard deviation of each measure. For secondary outcomes related to HIV and HCV, the sample size also provides $80 \%$ power to find a twotailed difference in proportion screened for $\mathrm{HIV} / \mathrm{HCV}$ of 16\% [72] (conservatively assuming that screening for one group nears $50 \%$, the point requiring the largest difference to achieve a specified power).

Mediation and moderation analyses. Power for detecting specific parameter changes in the structural model will be estimated using a procedure proposed by Satorra and Saris [73] that approximates the noncentral chi-square distribution. A total $N$ of approximately 220 patients would provide adequate power $(>.80)$ to detect a group difference in a parameter by 0.4 standard deviations (a moderate effect). Since our primary analysis projects a final $N$ of 286, we are confident our secondary process analysis will have adequate power.

Missing data. In previous addiction work, we completed $85 \%$ of surveys at 4,8 , and 12 months. We anticipate greater reductions at months 16,20 , and 24 , reaching about $65 \%$ by month 24 . In previous studies, we have kept missing data on core items in a survey to about $2 \%$ and expect a comparable rate in this study. In addiction treatment, data are not likely to be missing at random (i.e., the probability that data are missing is related to what the data would have been had the data been observed). For example, some patients may not want to disclose opioid use in surveys. Because this may lead to biased parameter estimates for our models, we will identify missing data patterns and use pattern-mixture modeling to test the sensitivity of our longitudinal intervention analysis to missing data assumptions [74-77] and conduct other sensitivity analyses after imputing missing data with a range of clinically plausible values based on explicit assumptions for the missing data (e.g., best-case, worst-case; with and without multiple imputation) [78-80].

Intention-to-treat and subject noncompliance. Standard intention-to-treat (ITT) estimates the average treatment effect by comparing outcomes based on assignment to treatment, but ignoring use of the treatment. Because ITT estimates do not represent treatment efficacy under noncompliance (e.g., a patient is randomized to A-CHESS but does not use the system), we will address noncompliance by also estimating treatment effects only for compliers using as-treated, per-protocol [81], and CACE (Complier Average Causal Effect) [82].

Dropout rates. In our A-CHESS RCT with alcoholdependent patients [33], 88 patients were using opioids as well as alcohol; 261 were not using opioids. We compared the post-test survey response rate of opioid-using patients to the response rate of patients who did not use opioids. The non-opioid-using patients' response rates were $94.3 \%$ at 4 months; $90.6 \%$ at 8 months, and $86.7 \%$ at 12 months. The opioid-using patients' response rates were $91.2 \%, 86 \%$, and $79.1 \%$. Response rates declined in a relatively linear fashion in both groups, with reductions of about 5 percentage points in each period. We assumed a $65 \%$ response rate at 24 months by continuing the drop off rate for each of the three periods from $79 \%$ to $74 \%$ to $69 \%$ to $64 \%$. Hence, we believe it is likely that by the end of the study we will still be able to reach $65 \%$ of patients originally enrolled.

Mediation analysis. Mediator variables will all be collected in the first two visits (at the 4- and 8-month visits) while the outcome (illicit drug use days) will be collected at months 12, 16, 20, and 24. Because mediator-outcome relations might reflect the effects of drug use while the mediator is being assessed (e.g., drug use might suppress ratings of competence), drug use that occurs during the mediator assessment period will be covaried out of models to examine and control its influence. Moreover, to assess the nonorthogonality of the mediators (which seems likely with the selfdetermination variables), we will use multiple mediator analyses based on a Bayesian approach illustrated in Yuan and MacKinnon [83]. This Bayesian estimation of the meditational models can be implemented through Markov Chain Monte Carlo (MCMC) techniques. Unlike more traditional estimation methods, such as maximum likelihood or least squares methods, for example, MCMC methods rely on sampling techniques to estimate model parameters and resulting mediation effects (i.e., iterative sampling from the parameter distributions is used to estimate confidence intervals to identify significant effects). An appealing feature of the method is its relative ease of implementation, particularly for complex statistical models. Similar to Yuan and MacKinnon, we will implement MCMC using WinBUGS 1.4 [84]. The multiple mediator models will be conducted with only those mediators shown to be significant in univariate models. See Bolt et al. [85] for our previous application of this analytic approach. 
Qualitative analysis. Content analysis [86] of interview transcripts will describe the role that MAT + A-CHESS plays in sustaining opioid recovery and reducing HIV/ $\mathrm{HCV}$ risk; identify potential improvements in MAT and A-CHESS; and supplement the quantitative analysis. UW research staff will: (1) construct a coding scheme [87] by combining categories flowing from the research questions, categories used in previous studies, and a preliminary examination of the data. Ideas will be our unit of analysis (rather than words or paragraphs) to capture references to a concept as well as direct statements about it, (2) test the coding scheme on a sample of data. Three coders will independently code the data in NVivo. We will calculate intercoder reliability and develop a set of coding instructions to insure reliability of at least .80 , (3) code the full dataset and create a conceptual model to help explain the mechanisms by which, and conditions under which, the interventions affect opioid use and HIV/HCV screening. These conclusions will help us understand the benefits of and modifications needed for MAT + A-CHESS (and MAT alone) to sustain recovery over the long term.

\section{Discussion}

This study is the first to our knowledge to test whether MAT for OUDs, when combined with a smartphonebased relapse prevention system, can significantly improve long-term recovery from opioid dependence when compared with MAT alone. The study will also explore for whom, and under what circumstances, A-CHESS does and does not work, and whether the tested bundle of services can reduce relapse-related health service use.

We believe that the HIV/HCV component of the study adds value to the intervention in two ways: (1) the prevalence of $\mathrm{HIV} / \mathrm{HCV}$ infection is high among opioidusing populations, yet most addiction treatment centers do not perform routine testing; bundling HIV/HCV services with A-CHESS could improve screening rates in a high-risk population for two serious but highly treatable conditions, (2) screening for $\mathrm{HIV} / \mathrm{HCV}$ is consistent with the project's overall goal of improving access to comprehensive health services for opioid-dependent patients, rather than focusing narrowly on promoting abstinence from opioids. We recognize that, despite the availability of evidence-based interventions, many patients who have injected opioids will relapse. The bundled intervention seeks to meet a public health goal of reducing the number of people who are infected with HIV or HCV but are unaware of being infected and, therefore, continue to place others at risk.

\section{Public health impact}

mHealth systems can attend to patients nearly as constantly as addiction does. At the end of this project, we will understand whether bundling MAT with an mHealth relapse prevention system can improve longterm recovery from opioid dependence. Just as important, we will better understand factors that will improve the design and delivery of treatment. This new knowledge could have wide and lasting benefits for patients who suffer from SUDs and other chronic conditions and for the health systems designed to help them.

\section{Trial status}

The trial has received ethical approval and recruited 23 participants to date (8 June 2016). We anticipate ending recruitment in February 2018.

\section{Additional files}

Additional file 1: Completed SPIRIT Checklist, the addendum to which contains the completed WHO Checklist. (DOC 148 kb)

Additional file 2: Completed SPIRIT figure. (PDF $103 \mathrm{~kb}$ )

\section{Abbreviations}

A-CHESS: Addiction CHESS (Comprehensive Health Enhancement Support System); BAM: Brief Addiction Monitor; DSM-V: Diagnostic and statistical manual of mental disorders, fifth edition; HCV: Hepatitis C virus; HIV: Human immunodeficiency virus; MAT: Medication-assisted treatment; NA/AA: Narcotics Anonymous, Alcoholics Anonymous; OUD: Opioid use disorder; SUD: Substance use disorder

\section{Acknowledgements}

The authors also wish to thank the people who are making the research possible: Nancy Paull, Lisa Garcia, Robin Quinterno, Claudio DosSantos, and Pat Affonso at Stanley Street Treatment and Resources (Fall River,

Massachusetts); Raymond Tamasi and Kelly McCarthy at Gosnold on Cape Cod (Falmouth, Massachusetts); and Norman Briggs and Laura Fabick at ARC Community Services (Madison, Wisconsin). In addition, the authors thank the project staff and tech team members at CHESS: Adam Maus, Julie Judkins, Samyuktha Anumolu, Matthew Wright, Patrick Rogne, Kristina Fischer, and Cameron Hall, as well as the patients and health care practitioners who have helped us understand opioid dependence and develop an approach that may help relieve it.

\section{Funding}

The National Institute on Drug Abuse (NIDA) is funding the study (1R01DA040449-01). The funder has no role in the design of the study; the collection, analysis, and study design, the interpretation of data, or the publication of results.

\section{Availability of data and materials}

The datasets and materials used and/or analyzed during the current study will be available from the first author (DHG Sr.) on reasonable request.

\section{Authors' contributions}

DHG Sr. and DS designed the study. DHG Sr. drafted the original manuscript. GL, FM, MLM, RAJ, RK, RPW, AQ, EA, DHG Jr., KP, and CT contributed to the design and conduct of the study. DHG Sr., GL, RAJ, and RK made critical revisions to the manuscript. All authors read, contributed to, and approved the final manuscript.

\section{Competing interests}

Authors Gustafson, McTavish, Johnson, and Quanbeck have a shareholder interest in CHESS Mobile Health, a small business that develops web-based health care technology for patients and family members. This relationship is extensively managed by the authors and the University of Wisconsin. All other authors declare that they have no competing interests. 


\section{Consent for publication}

Not applicable.

\section{Ethical approval and consent to participate}

The study received approval from the Health Sciences Institutional Review Board at the University of Wisconsin-Madison (\#2015-1418) and the Western Institutional Review Board in Puyallup, Washington (\#1163410). We will obtain informed consent from all participants in the study.

\section{Author details}

${ }^{1}$ Center for Health Enhancement Systems Studies, University of Wisconsin-Madison, Madison, WI 53706, USA. ${ }^{2}$ School of Journalism and Mass Communication, University of Wisconsin-Madison, Madison, WI 53706, USA. ${ }^{3}$ Communication Arts Department, University of Wisconsin-Madison, Madison, WI 53706, USA. ${ }^{4}$ Department of Medicine, University of Wisconsin School of Medicine and Public Health, Madison, Wisconsin 53705, USA.

${ }^{5}$ Access Community Health Centers, Madison WI 53715, USA. ${ }^{6}$ Mass Communication Research Center, School of Journalism and Mass Communication, University of Wisconsin-Madison, Madison, WI 53706, USA.

\section{Received: 14 June 2016 Accepted: 23 November 2016} Published online: 12 December 2016

\section{References}

1. Substance Abuse and Mental Health Services Administration (SAMHSA). Results from the 2012 National Survey on Drug Use and Health: Summary of National Findings. Rockville: Substance Abuse and Mental Health Services Administration; 2013.

2. Volkow N, Director, National Institute on Drug Abuse (NIDA). America's addiction to opioids: heroin and prescription drug abuse. Washington, DC: National Institute on Drug Abuse (NIDA), National Institutes of Health (NIH); 2014.

3. Substance Abuse and Mental Health Services Administration (SAMHSA) Drug Abuse Warning Network, 2007: national estimates of drug-related emergency department visits. Rockville: Substance Abuse and Mental Health Services Administration; 2008.

4. News from the Centers for Disease Control and Prevention (CDC). Opioid overdoses continue to climb. JAMA. 2016;315(6):550.

5. Conrad C, Bradley HM, Broz D, Buddha S, Chapman EL, Galang RR, et al. Community outbreak of HIV infection linked to injection drug use of oxymorphone-Indiana, 2015. MMWR. 2015;64(16):443-4.

6. Zibbell JE, lqbal K, Patel RC, Suryaprasad A, Sanders KJ, Moore-Moravian L, et al. Increases in hepatitis $C$ virus infection related to injection drug use among persons aged $\leq 30$ years - Kentucky, Tennessee, Virginia, and West Virginia, 2006-2012. MMWR. 2015;64(17):453-8.

7. Kleber HD. Pharmacologic treatments for opioid dependence: detoxification and maintenance options. Dialogues Clin Neurosci. 2007;9(4):455.

8. Heron KE, Smyth JM. Ecological momentary interventions: incorporating mobile technology into psychosocial and health behaviour treatments. Br J Health Psychol. 2010;15(Pt 1):1-39.

9. Center for Substance Abuse Treatment (CSAT). Medication-assisted treatment for opioid addiction in opioid treatment programs. Rockville: U.S. Department of Health and Human Services (DHHS); 2005.

10. Hendershot CS, Witkiewitz K, George WH, Marlatt GA. Relapse prevention for addictive behaviors. Subst Abuse Treat Prev Policy. 2011;6:17.

11. Substance Abuse and Mental Health Services Administration (SAMHSA). 2012 National Survey on Drug Use and Health: detailed tables - 5.1 to 5.56. Rockville: Substance Abuse and Mental Health Services Administration, Center for Behavioral Health Statistics and Quality; 2012.

12. Brady KT, Back SE, Greenfield SF. Women and addiction: a comprehensive handbook. New York: Guilford Press; 2009

13. Simpson DD, Joe GW, Brown BS. Treatment retention and follow-up outcomes in the Drug Abuse Treatment Outcome Study (DATOS). Psychol Addict Behav. 1997;11(4):294.

14. Brooks AC, Comer SD, Sullivan MA, Bisaga A, Carpenter KM, Raby WM, et al. Long-acting injectable versus oral naltrexone maintenance therapy with psychosocial intervention for heroin dependence: a quasi-experiment. J Clin Psychiatry. 2010;71(10):1371-8.

15. Fiellin DA, Pantalon MV, Chawarski MC, Moore BA, Sullivan LE, O'Connor PG, et al. Counseling plus buprenorphine-naloxone maintenance therapy for opioid dependence. N Engl J Med. 2006;355(4):365-74.
16. Witkiewitz K, Marlatt GA. Relapse prevention for alcohol and drug problems: that was Zen, this is Tao. Am Psychol. 2004;59(4):224-35.

17. McKay JR, Weiss RV. A review of temporal effects and outcome predictors in substance abuse treatment studies with long-term follow-ups preliminary results and methodological issues. Eval Rev. 2001;25(2):113-61.

18. McLellan A. The outcomes movement in substance abuse treatment: comments, concerns and criticisms. In: Sorenson JL, Rawson RA, Guydish A, Zweben JE, editors. Drug abuse treatment through collaboration: practice and research partnerships that work. Washington, DC: American Psychological Association; 2002. p. 119-34.

19. Bradizza CM, Stasiewicz PR, Paas ND. Relapse to alcohol and drug use among individuals diagnosed with co-occurring mental health and substance use disorders: a review. Clin Psychol Rev. 2006;26(2):162-78.

20. Zhang Z, Friedmann PD, Gerstein DR. Does retention matter? Treatment duration and improvement in drug use. Addiction. 2003;98(5):673-84.

21. Giordano TP, Gifford AL, White Jr AC, Suarez-Almazor ME, Rabeneck L, Hartman C, et al. Retention in care: a challenge to survival with HIV infection. Clin Infect Dis. 2007;44(11):1493-9.

22. Torian LV, Wiewel EW, Liu KL, Sackoff JE, Frieden TR. Risk factors for delayed initiation of medical care after diagnosis of human immunodeficiency virus. Arch Intern Med. 2008;168(11):1181-7.

23. Fleishman JA, Yehia BR, Moore RD, Korthuis PT, Gebo KA. Establishment, retention, and loss to follow-up in outpatient HIV care. J Acquir Immune Defic Syndr. 2012;60(3):249-59.

24. Schepens T, Morreel S, Florence E, Koole O, Colebunders R. Incidence and risk factors associated with lost to follow-up in a Belgian cohort of HIVinfected patients treated with highly active antiretroviral therapy. Int J STD AIDS. 2010;21(11):765-9.

25. Torian LV, Wiewel EW. Continuity of HIV-related medical care, New York City, 2005-2009: do patients who initiate care stay in care? AIDS Patient Care STDS. 2011;25(2):79-88.

26. Westergaard RP, Hess T, Astemborski J, Mehta SH, Kirk GD. Longitudinal changes in engagement in care and viral suppression for HIV-infected injection drug users. AIDS. 2013;27(16):2559-66.

27. Westergaard RP, Ambrose BK, Mehta SH, Kirk GD. Provider and clinic-level correlates of deferring antiretroviral therapy for people who inject drugs: a survey of North American HIV providers. J Int AIDS Soc. 2012;15(1):10.

28. Hanna DB, Buchacz K, Gebo KA, Hessol NA, Horberg MA, Jacobson LP, et al. Trends and disparities in antiretroviral therapy initiation and virologic suppression among newly treatment-eligible HIV-infected individuals in North America, 2001-2009. Clin Infect Dis. 2013;56(8):1174-82.

29. Weinbaum C, Lyerla R, Margolis H, Centers for Disease Control and Prevention. Prevention and control of infections with hepatitis viruses in correctional settings. Centers for Disease Control and Prevention. MMWR Recomm Rep. 2003;52(RR-1):1-36.

30. Amon JJ, Garfein RS, Ahdieh-Grant L, Armstrong GL, Ouellet LJ, Latka MH, et al. Prevalence of hepatitis $C$ virus infection among injection drug users in the United States, 1994-2004. Clin Infect Dis. 2008;46(12):1852-8.

31. Hagan H, Pouget ER, Des Jarlais DC, Lelutiu-Weinberger C. Meta-regression of hepatitis $C$ virus infection in relation to time since onset of illicit drug injection: the influence of time and place. Am J Epidemiol. 2008;168(10):1099-109.

32. Armstrong GL, Wasley A, Simard EP, McQuillan GM, Kuhnert WL, Alter MJ. The prevalence of hepatitis C virus infection in the United States, 1999 through 2002. Ann Intern Med. 2006;144(10):705-14.

33. Gustafson DH, McTavish FM, Chih MY, Atwood AK, Johnson RA, Boyle MG, et al. A smartphone application to support recovery from alcoholism: a randomized clinical trial. JAMA Psychiatry. 2014;71(5):566-72.

34. Mathews D, Evaluation Consultant. Evaluation Report: combatting addiction with technology for pregnant Appalachian women using smartphones. Hazard: Kentucky River Community Care, Inc; 2014.

35. Ryan RM, Deci EL. Self-determination theory and the facilitation of intrinsic motivation, social development, and well-being. Am Psychol. 2000;55(1):68-78.

36. Namkoong K, Shah DV, Han JY, Kim SC, Yoo W, Fan D, et al. Expression and reception of treatment information in breast cancer support groups: how health self-efficacy moderates effects on emotional well-being. Patient Educ Couns. 2010;81(Suppl):S41-7.

37. Ryan RM, Patrick H, Deci EL, Williams GC. Facilitating health behaviour change and its maintenance: interventions based on self-determination theory. Eur Health Psychol. 2008;10(1):2-5. 
38. Marlatt G, Gordon J. Relapse prevention: maintenance strategies in the treatment of addictive disorders. New York: Guilford Press; 1985.

39. Larimer ME, Palmer RS, Marlatt GA. Relapse prevention. An overview of Marlatt's cognitive-behavioral model. Alcohol Res Health. 1999;23(2):151-60.

40. Dennis ML, Lennox R, Scott C, Funk R. Comparing the ability of multiple measures of substance abuse treatment process to predict outcomes. Drug Alcohol Depend. 2014;140:e48.

41. Grossman P, Niemann L, Schmidt S, Walach H. Mindfulness-based stress reduction and health benefits: a meta-analysis. J Psychosom Res. 2004;57(1):35-43.

42. Nelson KG, Young $K$, Chapman $H$. Examining the performance of the Brief Addiction Monitor. J Subst Abuse Treat. 2014;46(4):472-81.

43. Voogt C, Kuntsche E, Kleinjan M, Poelen E, Engels R. Using ecological momentary assessment to test the effectiveness of a web-based brief alcohol intervention over time among heavy-drinking students: randomized controlled trial. J Med Internet Res. 2014;16(1):e5.

44. Krishna S, Boren SA, Balas EA. Healthcare via cell phones: a systematic review. Telemed J E Health. 2009:15(3):231-40.

45. Hawkins RP, Pingree S, Baker T, Roberts LJ, Shaw B, McDowell H, et al. Integrating eHealth with human services for breast cancer patients. Transl Behav Med. 2011;1(1):146-54.

46. Quanbeck AR, Gustafson DH, Marsch LA, McTavish F, Brown RT, Mares ML, et al. Integrating addiction treatment into primary care using mobile health technology: protocol for an implementation research study. Implement Sci. 2014;9:65.

47. Harris PA, Taylor R, Thielke R, Payne J, Gonzalez N, Conde JG. Research electronic data capture (REDCap) — a metadata-driven methodology and workflow process for providing translational research informatics support. J Biomed Inform. 2009;42(2):377-81.

48. Keen J. Case studies. In: Pope C, Mays N, editors. Qualitative research in healthcare. London: Wiley; 2008. p. 112-20.

49. Yin RK. Case study research: design and methods. 5th ed. Los Angeles: Sage Publications; 2014

50. Baker GR. The contribution of case study research to knowledge of how to improve quality of care. BMJ Qual Saf. 2011;20 Suppl 1:i30-5.

51. Hjermstad MJ, Fayers PM, Haugen DF, Caraceni A, Hanks GW, Loge JH, et al. Studies comparing Numerical Rating Scales, Verbal Rating Scales, and Visual Analogue Scales for assessment of pain intensity in adults: a systematic literature review. J Pain Symptom Manage. 2011;41(6):1073-93.

52. Donovan DM, Bigelow GE, Brigham GS, Carroll KM, Cohen AJ, Gardin JG, et al. Primary outcome indices in illicit drug dependence treatment research: systematic approach to selection and measurement of drug use end-points in clinical trials. Addiction. 2012;107(4):694-708.

53. Sobell LC, Brown J, Leo Gl, Sobell MB. The reliability of the Alcohol Timeline Followback when administered by telephone and by computer. Drug Alcohol Depend. 1996;42(1):49-54.

54. Sobell LC, Maisto SA, Sobell MB, Cooper AM. Reliability of alcohol abusers' self-reports of drinking behavior. Behav Res Ther. 1979;17(2):157-60.

55. Cacciola JS, Alterman Al, Dephilippis D, Drapkin ML, Valadez Jr C, Fala NC, et al. Development and initial evaluation of the Brief Addiction Monitor (BAM). J Subst Abuse Treat. 2013;44(3):256-63.

56. Ferreira-Valente MA, Pais-Ribeiro $J \mathrm{~L}$, Jensen MP. Validity of four pain intensity rating scales. Pain. 2011;152(10):2399-404.

57. Darke S, Hall W, Heather N, Ward J, Wodak A. The reliability and validity of a scale to measure HIV risk-taking behaviour among intravenous drug users. AIDS. 1991;5(2):181-6.

58. Diener E, Emmons RA, Larsen RJ, Griffin S. The Satisfaction with Life Scale. J Pers Asses. 1985;49(1):71-5.

59. Morisky DE, Ang A, Krousel-Wood M, Ward HJ. Predictive validity of a medication adherence measure in an outpatient setting. J Clin Hypertens (Greenwich). 2008;10(5):348-54.

60. Williams GC, Cox EM, Kouides R, Deci EL. Presenting the facts about smoking to adolescents: effects of an autonomy-supportive style. Arch Pediatr Adolesc Med. 1999;153(9):959-64.

61. Sklar SM, Annis HM, Turner NE. Development and validation of the DrugTaking Confidence Questionnaire: a measure of coping self-efficacy. Addict Behav. 1997;22(5):655-70

62. Watson D, Clark LA, Tellegen A. Development and validation of brief measures of positive and negative affect: the PANAS scales. J Pers Soc Psychol. 1988;54(6):1063.

63. Measelle JR, Stice E, Springer DW. A prospective test of the negative affect model of substance abuse: moderating effects of social support. Psychol Addict Behav. 2006;20(3):225.
64. Luoma JB, Nobles RH, Drake CE, Hayes SC, O'Hair A, Fletcher L, et al. Selfstigma in substance abuse: development of a new measure. J Psychopathol Behav Assess. 2013;35(2):223-34.

65. McCollister KE, French MT. The relative contribution of outcome domains in the total economic benefit of addiction interventions: a review of first findings. Addiction. 2003;98(12):1647-59.

66. Weinick RM, Bristol SJ, DesRoches CM. Urgent care centers in the US: findings from a national survey. BMC Health Serv Res. 2009;9(1):79.

67. French MT, Popovici I, Tapsell L. The economic costs of substance abuse treatment: updated estimates and cost bands for program assessment and reimbursement. J Subst Abuse Treat. 2008;35(4):462-9.

68. Bell J, Shanahan M, Mutch C, Rea F, Ryan A, Batey R, et al. A randomized trial of effectiveness and cost-effectiveness of observed versus unobserved administration of buprenorphine-naloxone for heroin dependence. Addiction. 2007:102(12):1899-907.

69. Hedeker D, Gibbons RD, Waternaux C. Sample size estimation for longitudinal designs with attrition: comparing time-related contrasts between two groups. J Educ Behav Stat. 1999;24(1):70-93.

70. Cohen J. Statistical power analysis for the behavioral sciences. 2nd ed. Hillsdale: Lawrence Erlbaum Associates; 1988.

71. McKay JR, Lynch KG, Shepard DS, Ratichek S, Morrison R, Koppenhaver J, et al. The effectiveness of telephone-based continuing care in the clinical management of alcohol and cocaine use disorders: 12-month outcomes. J Consult Clin Psychol. 2004;72(6):967-79.

72. Hintze J. PASS 11. Kaysville: NCSS; 2011.

73. Satorra A, Saris WE. Power of the likelihood ratio test in covariance structure analysis. Psychometrika. 1985;50(1):83-90.

74. Diggle $P$, Kenward MG. Informative drop-out in longitudinal data analysis. Appl Stat. 1994;43:49-93.

75. Hedeker D, Gibbons RD. Application of random-effects pattern-mixture models for missing data in longitudinal studies. Psychol Methods. 1997;2(1): 64.

76. Hedeker D, Gibbons RD. Longitudinal data analysis. Hoboken: Wiley; 2006.

77. Enders CK. Missing not at random models for latent growth curve analyses. Psychol Methods. 2011:16(1):1-16.

78. Birnbaum HG, White AG, Schiller M, Waldman T, Cleveland JM, Roland CL. Societal costs of prescription opioid abuse, dependence, and misuse in the United States. Pain Med. 2011;12(4):657-67.

79. Hedeker D, Mermelstein RJ, Demirtas H. Analysis of binary outcomes with missing data: missing = smoking, last observation carried forward, and a little multiple imputation. Addiction. 2007;102(10):1564-73.

80. Thabane L, Mbuagbaw L, Zhang S, Samaan Z, Marcucci M, Ye C, et al. A tutorial on sensitivity analyses in clinical trials: the what, why, when and how. BMC Med Res Methodol. 2013:13:92.

81. Little RJ, Yau LHY. Statistical techniques for analyzing data from prevention trials: treatment of no-shows using Rubin's causal model. Psychol Methods. 1998;3(2):147

82. Jo B. Statistical power in randomized intervention studies with noncompliance. Psychol Methods. 2002;7(2):178-93.

83. Yuan Y, MacKinnon DP. Bayesian mediation analysis. Psychol Methods. 2009;14(4):301-22

84. Spiegelhalter DJ. Understanding uncertainty. Ann Fam Med. 2008;6(3):196-7.

85. Bolt DM, Piper ME, Theobald WE, Baker TB. Why two smoking cessation agents work better than one: role of craving suppression. J Consult Clin Psychol. 2012;80(1):54-65.

86. Mayring P. Qualitative content analysis. In: Flick U, Kardoff EV, Steinke I, editors. A companion to qualitative research. Thousand Oaks: Sage; 2004. p. 266-9.

87. Berelson B. Content analysis in communication research. New York: Free Press; 1952 